\title{
Criminologie
}

\section{Lorsque le crime rapporte}

Les apprentissages réalisés dans des activités criminelles mobilisés dans le processus d'insertion professionnelle Benefits of crime Learnings aquired through criminal activities incorporated
into the professionnal integration process

Cuando el crimen paga Los aprendizajes realizados en actividades criminales
movilizados en el proceso de inserción profesional

\section{Marie-Michelle Pariseau et Eddy Supeno}

Volume 52, numéro 2, automne 2019

URI : https://id.erudit.org/iderudit/1065863ar

DOI : https://doi.org/10.7202/1065863ar

Aller au sommaire du numéro

Éditeur(s)

Les Presses de l’Université de Montréal

ISSN

0316-0041 (imprimé)

1492-1367 (numérique)

Découvrir la revue

Citer cet article

Pariseau, M.-M. \& Supeno, E. (2019). Lorsque le crime rapporte : les apprentissages réalisés dans des activités criminelles mobilisés dans le processus d'insertion professionnelle. Criminologie, 52(2), 239-266. https://doi.org/10.7202/1065863ar

\section{Résumé de l'article}

Les activités criminelles font partie de la catégorie des activités de la vie sociale sur lesquelles pèse une certaine stigmatisation. Des recherches en criminologie ont cependant démontré que les personnes qui exercent ces activités en retirent des bénéfices : gains financiers, reconnaissance symbolique et apprentissages variés. Si des écrits exposent les apprentissages réalisés pour pouvoir exercer ces activités criminelles, peu d'écrits se penchent sur les apprentissages réalisés dans la pratique de ces activités. Or, certains de ces apprentissages constitueraient des compétences socialement valorisées sur le plan de l'employabilité, n'eût été le jugement social pesant sur elles. Ici, des apprentissages réalisés dans l'exercice d'activités criminelles mobilisés dans le processus d'insertion professionnelle offriraient une compréhension plus ajustée de la complexité du processus d'insertion professionnelle, notamment auprès de jeunes adultes, diplômés ou non. Cet article présente les résultats d'une recherche qualitative menée auprès de cinq jeunes adultes ayant exercé des activités criminelles pour en voir les apprentissages mobilisés ou susceptibles de l'être dans leur processus d'insertion professionnelle dans un emploi légal. 


\title{
Lorsque le crime rapporte Les apprentissages réalisés dans des activités criminelles mobilisés dans le processus d'insertion professionnelle
}

\author{
Marie-Michelle Pariseau ${ }^{1}$ \\ Conseillère d'orientation \\ Membre professionnelle associée \\ Centre d'études et de recherches sur les transitions et l'apprentissage (CÉRTA) \\ Faculté d'éducation, Université de Sherbrooke \\ marie.michelle.pariseau@usherbrooke.ca \\ Eddy Supeno \\ Professeur agrégé \\ Conseiller d'orientation \\ Membre régulier \\ Centre d'études et de recherches sur les transitions et l'apprentissage (CÉRTA) \\ Faculté d'éducation, Université de Sherbrooke \\ eddy.supeno@usherbrooke.ca
}

RÉSUMÉ - Les activités criminelles font partie de la catégorie des activités de la vie sociale sur lesquelles pèse une certaine stigmatisation. Des recherches en criminologie ont cependant démontré que les personnes qui exercent ces activités en retirent des bénéfices: gains financiers, reconnaissance symbolique et apprentissages variés. Si des écrits exposent les apprentissages réalisés pour pouvoir exercer ces activités criminelles, peu d'écrits se penchent sur les apprentissages réalisés dans la pratique de ces activités. Or, certains de ces apprentissages constitueraient des compétences socialement valorisées sur le plan de l'employabilité, n'eût été le jugement social pesant sur elles. Ici, des apprentissages réalisés dans l'exercice d'activités criminelles mobilisés dans le processus d'insertion professionnelle offriraient une compréhension plus ajustée de la complexité du processus d'insertion professionnelle, notamment auprès de jeunes adultes, diplômés ou non. Cet article présente les résultats d'une recherche qualitative menée auprès de cinq jeunes adultes ayant exercé des activités criminelles pour en voir les apprentissages mobilisés ou susceptibles de l'être dans leur processus d'insertion professionnelle dans un emploi légal.

1. Centre d'études et de recherches sur les transitions et l'apprentissage (CÉRTA), Université de Sherbrooke, Faculté d'éducation, 2500, boul. de l'Université, local A1-238, Sherbrooke (Québec), Canada, J1K 2R1.

Criminologie, vol. 52, nº 2 (2019) 
MOTS CLÉS - Jeunes adultes, apprentissages, criminalité, insertion professionnelle, théorie de l'activité.

\section{Introduction}

Bien que les jeunes adultes constituent un bassin de main-d'œuvre potentiel en raison notamment du vieillissement de la population québécoise (Gouvernement du Québec, 2011), ils ne se voient pas garantis d'une place sur le marché du travail. Cela est d'autant plus vrai pour ceux ayant un casier judiciaire. Selon le Comité consultatif clientèle judiciarisée adulte (2017), le casier constitue en effet un obstacle majeur qui limite l'accès au marché du travail. Par ailleurs, l'absence de qualifications ${ }^{2}$ reconnues peut souvent - mais pas systématiquement (Vultur, Trottier et Gauthier, 2002) - constituer un obstacle à l'intégration professionnelle lorsque «la formation et les qualifications sont bien souvent considérées comme un critère d'embauche incontournable» (Champagne, Malenfant, Bellemare et Briand, 2013, p. 187).

Dans ce contexte, pour certains jeunes adultes, une stratégie compensatoire parmi d'autres consiste à s'investir, temporairement ou non, dans des activités criminelles lorsque l'accès à des emplois légaux s'avère difficile. Les conditions de travail et la précarité en emploi agissent parfois comme un puissant incitatif à déserter complètement le marché officiel du travail et à adhérer au «capitalisme de butin» de la rue où, en s'intégrant à des gangs et au commerce de la drogue, ils arrivent au moins à sauvegarder un sens viril de l'honneur, à maintenir le respect d'eux-mêmes, et même à nourrir certains espoirs de richesse économique (McCarthy et Hagan, 2004, p. 144).

Car au-delà de la dangerosité liée aux activités criminelles, certains jeunes adultes peuvent en retirer des bénéfices potentiels (Charette, 2010; Morselli et Tremblay, 2004). Tremblay (2010) fait état d'une littérature abondante portant sur la réussite et la performance criminelle. Plusieurs écrits documentent la criminalité comme une stratégie pour obtenir des gains illicites ou subvenir à des besoins (Charest et Tremblay, 2009; McCarthy et Hagan, 2001, 2004; Morselli et Tremblay, 2004; Robitaille, 2004; Uggen et Thompson, 2003). On sait également que l'implication dans la criminalité peut être une source de reconnaissance symbolique en répondant à des besoins identitaires

2. Le terme «qualifications» fait référence aux apprentissages formels réalisés au sein d'une institution scolaire. 
d'épanouissement social et psychologique lorsque l'économie formelle, pourvoyeuse d'emplois légaux, ne le permet pas (Bureau et Fendt, 2010). Côté, Blais, Bellot et Manseau (2013) indiquent que ces activités criminelles peuvent devenir un véritable levier d'insertion sociale et offrir notamment une image sociale d'une certaine réussite. Finalement, l'exercice d'activités criminelles permettrait de développer des apprentissages variés et diverses compétences (McCarthy et Hagan, 2001; McCarthy, Hagan et Cohen, 1998). Posséder des compétences criminelles influencera positivement les retombées monétaires et sociales liées à l'exercice d'activités criminelles (Tremblay et Morselli, 2000). Si plusieurs auteurs se sont penchés sur les causes de l'implication et la poursuite d'activités criminelles (Lanctôt, 2005; Le Blanc, 2010; Ouellet, 2012; Vitaro, Brendgen, Ladouceur et Tremblay, 2001 ; Warr, 1998), d'autres ont examiné la manière dont les individus apprennent à exercer des activités criminelles: parfois par mentorat (Adler et Adler, 1983; Morselli, Tremblay et McCarthy; 2006) ou par imitation par exemple (Akers et Jennings, 2009; Becker, 1985; Sutherland, 1966). Il est possible d'établir certaines similarités entre le capital humain ou social $^{3}$ et le capital criminel (Loughran, Nguyen, Piquero et Fagan, 2013). Ainsi, l'influence des pairs dans l'exercice d'activités criminelles permettrait le développement des savoirs et de compétences propices à favoriser le succès criminel (McCarthy et Hagan, 2001 ; McCarthy et al., 1998). Pour Uggen et Thompson (2003), les individus qui exercent des activités criminelles «acquièrent des compétences et de l'expérience, reçoivent une tutelle informelle qui peut prendre la forme de gains illicites» (p. 148). Letkemann (1973) présente plus spécifiquement trois formes d'apprentissage nécessaires dans la réalisation des cambriolages: ceux mécaniques (les outils et la procédure à suivre), ceux organisationnels (la planification et l'exécution de l'activité) et ceux sociaux (contrôler les formes de tensions et composer avec les victimes). Adler et Adler (1983) précisent que connaître une certaine réussite dans l'importation de drogues exige des savoirs, de l'expérience et un réseau de contacts. Selon Roy et Hurtubise (2004), l'exercice d'activités telles que la vente de drogue permet de développer des compétences variées, dont l'«encadrement et formation de la main-d'œuvre (pusher et squeegee), mode de

3. Selon Morselli et al. (2006), le capital social fait référence aux ressources potentielles dans les relations interpersonnelles. Ces auteurs précisent par ailleurs que le capital social est essentiel au succès dans des occupations particulières, notamment en ce qui concerne les activités criminelles. 
surveillance des activités, contrôle de la qualité des produits (la drogue doit être de bonne qualité pour maintenir le marché), constitution de réseaux (pour écouler la marchandise ou donner les services)» (p. 134). Selon Bureau et Fendt (2010), la réussite d'une personne trafiquante d'armes ou de drogue nécessite le développement de nombreux savoirs entrepreneuriaux, beaucoup de patience, de travail et de talent. Ce bref état des connaissances montre que l'exercice d'activités criminelles est susceptible de conduire à plusieurs apprentissages ou d'en exiger l'acquisition pour la personne, au point d'y développer une certaine compétence, voire y connaître une certaine réussite. Plus précisément, les apprentissages en question pourraient, à bien des égards, être pertinents et même socialement valorisés sous l'angle de l'employabilité et de l'insertion professionnelle.

Or, les apprentissages réalisés dans l'exercice d'activités criminelles ne semblent pas portés à être reconnus formellement, car le marché du travail demande généralement une attestation officielle des qualifications à l'emploi (Morselli et Tremblay, 2004). Selon Roy et Hurtubise (2004), la non-reconnaissance de ces activités est due «en raison de barrières culturelles, légales, morales, institutionnelles et politiques» (p. 128) ${ }^{4}$. Cependant, peu d'écrits s'intéressent formellement à la mobilisation d'apprentissages réalisés dans des activités criminelles en contexte de recherche d'emploi. Pourtant, «celui qui brille par ses talents de revendeur de cocaïne devrait briller avec le même éclat dans la vente d'autres produits» (Morselli et Tremblay, 2004, p. 109). Par ailleurs, le Comité consultatif clientèle judiciarisée adulte (2014) rapporte que plusieurs problématiques rencontrées par les personnes judiciarisées concernent notamment des difficultés liées au marché du travail, aux compétences professionnelles (méconnaissance des techniques de recherche d'emploi, manque d'expérience dans les entrevues d'embauche, formation plus ou moins qualifiante, etc.) et au casier judiciaire (difficultés à se trouver un emploi, préjugés associés au casier judiciaire influençant l'insertion sociale, etc.). Si ces constats du Comité montrent que des compétences en matière d'insertion professionnelle semblent faire défaut aux personnes judiciarisées, notre état des connaissances documente, pour sa part, que ces personnes ne sont pas toutes

4. Pour Becker (1985), l'infraction aux règles données par un groupe social constitue la déviance et les individus qui transgressent ses règles sont étiquetés comme des outsiders. 
entièrement dépourvues de compétences potentiellement mobilisables sur le plan de l'employabilité.

Dans une perspective d'apprentissage tout au long de la vie (Delors, 1996), l'objectif de cette recherche est donc d'établir en quoi la mobilisation des apprentissages réalisés dans des activités criminelles peut participer ou non à la recherche d'emploi chez des personnes judiciarisées. Trois objectifs spécifiques ont été définis : 1) nommer et décrire les activités criminelles de jeunes adultes et les apprentissages qui y sont liés; 2) nommer et décrire les activités de recherche d'emploi de ces jeunes adultes; 3) cibler des apprentissages réalisés dans l'exercice d'activités criminelles mobilisés dans des activités liées à la recherche d'emploi.

Trois retombées sont anticipées dans cet article: a) contribuer à la littérature scientifique qui traite des retombées liées aux activités criminelles, s'agissant des apprentissages qui y sont développés; b) documenter la complexité du processus d'insertion professionnelle chez la population judiciarisée; c) proposer une piste potentielle d'intervention auprès de cette population sous l'angle de l'insertion professionnelle.

\section{Cadre d'analyse}

Au regard de l'objectif de recherche, le cadre s'articule autour de la théorie de l'activité d'Engeström (2001). Ses travaux abordent notamment l'importance du travail comme activité humaine dans un contexte d'équipe ou de collectif de travail (Dionne, 2015), ce qui est en cohérence avec Le Blanc (2003) quand il écrit que la «conduite délinquante a toujours été reconnue comme une activité de groupe» (p. 402). De plus, on peut appréhender les activités criminelles comme faisant partie de la sphère professionnelle (Bureau et Fendt, 2010), car certaines peuvent représenter des formes atypiques du travail (Roy et Hurtubise, 2004), voire être considérées comme des carrières criminelles (Becker, 1985; Blumstein et Cohen, 1987; Le Blanc, 1986; Piquero, Farrington et Blumstein, 2003). Par ailleurs, la théorie de l'activité d'Engeström permet de circonscrire conceptuellement les composantes des activités liées à la recherche d'emploi et de relever ainsi la mobilisation d'apprentissages d'une activité à l'autre. Décomposer deux systèmes d'activité à partir du même 
modèle conceptuel permet d'analyser la mobilisation des apprentissages d'un système à l'autre de manière uniforme et cohérente.

FIGURE 1

La structure du système de l'activité humaine d'Engeström

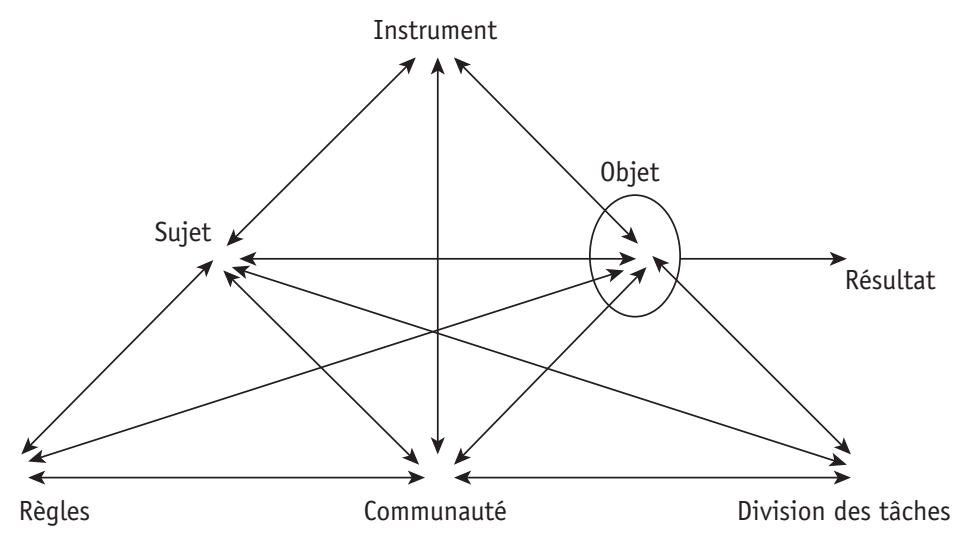

La modélisation du système d'activité selon Engeström

La modélisation du système d'activité d'Engeström (2001) est formée de six pointes qui s'interinfluencent entre elles (Figure 1). Le sujet est constitué de l'individu et de ses caractéristiques. Les instruments sont ceux utilisés par l'individu (le sujet) dans la réalisation de l'activité et prennent deux formes: matérielle ou symbolique. Les instruments matériels concernent des éléments tangibles, associés à des objets tels un téléphone ou une automobile. Les instruments symboliques renvoient aux éléments abstraits tels que le réseau de contacts et des notions jugées nécessaires à la réalisation de l'activité. Ces instruments symboliques peuvent renvoyer à la transmission des savoirs d'un individu qui est reconnu par son groupe de référence comme étant un «délinquant performant» pour en influencer d'autres (Tremblay, 2010). Les règles concernent les règlements, normes, valeurs et croyances véhiculés dans la société qui influencent la pratique de l'activité, comme le casier judiciaire par exemple. La communauté fait référence aux personnes qui vivent des événements similaires, qui partagent des expériences et des projets communs avec la personne (le sujet) dans la réalisation de 
l'activité. Comme l'avance la théorie de l'association différentielle (Sutherland, 1966), le comportement criminel est appris au contact d'autres personnes par un processus de communication, il s'apprend surtout à l'intérieur d'un groupe restreint de relations personnelles et, une fois adopté, il suppose que l'individu a) a acquis des techniques simples et complexes pour exercer l'activité criminelle et b) oriente ses raisonnements et ses attitudes en ce sens ${ }^{5}$. La division des tâches est la compartimentation des tâches dans la réalisation de l'activité6. L'interinfluence de ces cinq éléments mène à un sixième, l'objet, qui représente l'activité réalisée en soi. Finalement, l'activité produira des résultats. L'activité, orientée ou influencée par un but à atteindre, s'inscrit toujours dans un contexte social et est en relation avec d'autres systèmes d'activité. Les activités criminelles comme celles de recherche d'emploi peuvent être conceptuellement appréhendées, à l'image de toute autre activité, comme une série d'opérations effectuées à l'intérieur de cadres dans le but de réaliser un objectif.

Par ailleurs, cinq principes fondamentaux sous-tendent la théorie de l'activité pour Engeström $(2001)^{7}$ : premièrement, un système d'activité doit être en relation avec d'autres systèmes d'activité pour que celui-ci soit compréhensible. Deuxièmement, le système d'activité est composé de multiples points de vue, de traditions et d'intérêts, et possède ainsi sa propre historicité. Troisièmement, le système d'activité prend forme et se transforme sur une longue période de temps. Quatrièmement, des tensions dans l'activité auront pour effet de créer des contradictions ${ }^{8}$ qui contribuent au changement et au développement du système d'activité. Cinquièmement, lorsque les normes établies sont remises en question, et à partir de contradictions, une transformation expansive du système est réalisée 9 . Dès lors, l'objet et le motif de l'activité sont

5. Voir propositions 2, 3 et 4 de cette théorie (Sutherland, 1966).

6. Tremblay et Morselli (2000) indiquent que les pairs dans l'exercice d'activités criminelles agissent comme sources d'information et peuvent mener les individus à de meilleures opportunités criminelles et donc augmenter le capital criminel.

7. Si la culture et l'histoire partagées entre les membres et leur rôle dans la réalisation de l'activité sont des concepts centraux chez Engeström, plusieurs auteurs en criminologie abordent l'appropriation et la pratique d'actes criminels comme culturellement transmises par l'interaction entre les individus. À cet effet, voir notamment Cohen (1965).

8. Ce terme fait référence à des difficultés associées à la réalisation de l'activité en question.

9. Pour de plus amples informations sur la résolution de contradictions dans l'appropriation d'une nouvelle activité, se référer au cycle d'expansion, voir aussi Engeström et Sannino $(2011,2013)$. 
restructurés pour augmenter «l'horizon des possibilités» du modèle précédent.

Pour Engeström (2005), les individus apprennent en tout temps des choses qui ne sont pas nécessairement stables, définies et comprises à l'avance: «dans les transformations importantes dans nos vies personnelles [...], nous devons apprendre de nouvelles formes d'activités qui ne sont pas encore là. Elles sont littéralement apprises pendant qu'elles se créent» (Engeström, 2001, p. 138, notre traduction). Si plusieurs approches considèrent les savoirs - et l'expertise associée - de manière verticale, c'est-à-dire selon des échelons prédéterminés, une dimension horizontale est ici privilégiée pour comprendre et acquérir de l'expertise dans l'exercice d'une activité (Engeström, Engeström et Kärkkäinen, 1995). Une compréhension horizontale des savoirs signifie que l'individu - l'expert - fonctionne entre plusieurs contextes d'activité qui sont parallèles (Engeström, 2005). Le développement d'apprentissages s'effectue dans la réalisation même d'une activité, lorsque le sujet se retrouve devant des contradictions qu'il tente de résoudre; ce développement n'est donc pas linéaire ni prédéterminé et sera fortement influencé par le contexte dans lequel l'individu se situe.

\section{FIGURE 2}

Deux systèmes d'activité en interaction qui modélisent la troisième génération de la théorie de l'activité

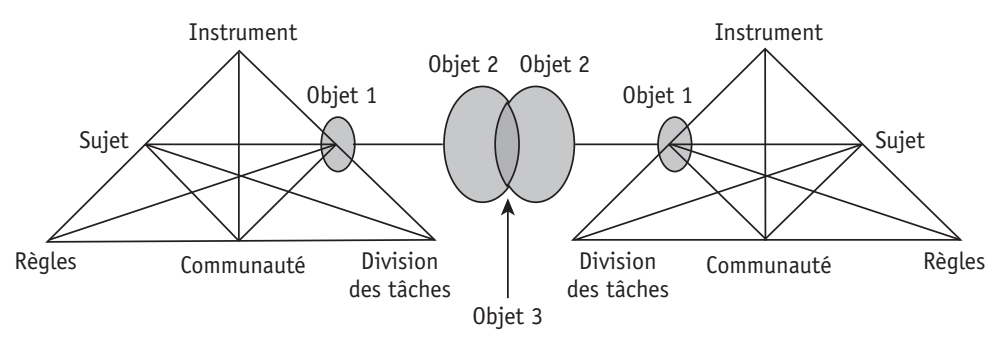

Afin de cibler les apprentissages d'une activité criminelle mobilisés vers une activité liée à la recherche d'emploi, le concept de déplacement transfrontalier $^{10}$ (Engeström et al., 1995) sera utilisé (Figure 2). Ce concept consiste à établir des interactions entre différentes activités (Bakx, Bakker, Koopman et Beijaard, 2016), lorsqu'une personne 
rencontre des contradictions dans la réalisation d'une activité pour laquelle elle n'est pas «qualifiée». La personne mobilisera des informations et des instruments jugés pertinents (qui deviennent des objets transfrontaliers) ${ }^{11}$ d'une autre activité dans laquelle elle se considère comme experte (ou en maitrise) pour tenter de résoudre les contradictions de l'activité qu'elle tente de s'approprier. Considérant notre objectif de recherche, l'individu peut alors mettre en relation des résultats liés aux activités criminelles - dans ce cas-ci des apprentissages - avec les résultats des activités liées à l'insertion en emploi, par exemple obtenir une entrevue. La négociation de ces résultats aboutit à une situation hybride, une autre forme de résultat, dans ce cas-ci, se trouver un emploi.

\section{Méthodologie}

Cette recherche mobilise un devis qualitatif en recourant à des entretiens semi-dirigés afin de rendre explicite la perspective des acteurs tout en accédant à leur expérience humaine (Savoie-Zajc, 2009). Être une personne âgée de plus de 18 ans, diplômée ou non du secondaire, avoir réalisé une ou plusieurs activités criminelles pour lesquelles elle a été jugée et résider au Québec constitue les critères d'inclusion de la recherche. Nous nous sommes adressés à plusieurs milieux pour le recrutement, dont des organismes d'aide à l'emploi et à l'intégration sociale dédiés aux jeunes adultes ou destinés aux personnes judiciarisées. Si certains n'ont pas donné suite à nos demandes, d'autres proposaient des personnes ne répondant pas aux critères d'inclusion. Nous avons maintenu l'échantillonnage volontaire et non probabiliste, mais avons alors choisi de recruter par réseaux (Fortin, 2010) par le site Facebook. Nous avons contacté différentes personnes qui interviennent auprès d'individus susceptibles de correspondre aux critères d'inclusion. Quatre individus ont ainsi été recrutés. Une maison de transition, intéressée à notre recherche, a permis de recruter un autre participant. L'échantillon est donc constitué de cinq personnes de sexe masculin âgées de 21 à 40 ans (moyenne d'âge: 27,6 ans). Un participant a arrêté après la première année du secondaire et un autre après la deuxième. Les trois autres participants ont terminé leurs études secondaires, dont deux des études professionnelles. La collecte de

11. Traduction libre de boundary objects. 
données s'est déroulée de décembre 2016 à mars 2017 sous forme d'entretiens semi-dirigés appuyés par un guide d'entretien et une compensation financière de 30 \$ était offerte à chaque participant. Le guide d'entretien est composé de cinq sections. La première section concerne les données sociodémographiques du participant. La deuxième section vise à déterminer et à décrire les activités liées à la recherche d'emploi ( $2^{\mathrm{e}}$ objectif spécifique). Des questions telles que "Qu'est-ce qui se passe exactement pour vous durant cette journée-là où vous êtes en recherche d'emploi?» ont été posées. La troisième section concerne le premier objectif spécifique qui est de nommer et de décrire les activités criminelles de jeunes adultes et les apprentissages qui y sont liés. La question «Parmi toutes les activités que vous avez nommées, y en a-t-il une ou plus d'une qui vous influence encore aujourd'hui? Si oui, laquelle ou lesquelles?» a été posée afin d'augmenter la possibilité que le participant choisisse une activité qui faciliterait les liens avec les activités liées à la recherche d'emploi. La quatrième section concerne le troisième objectif spécifique qui est de cibler des apprentissages réalisés dans l'exercice d'activités criminelles mobilisés dans des activités liées à la recherche d'emploi. Des questions telles que «Y a-t-il des choses que vous avez apprises durant l'activité X qui vous servent encore aujourd'hui? Si oui, lesquelles et de quelle manière vous servent-elles encore?» et «Quels liens pouvezvous faire entre les apprentissages que vous avez réalisés dans l'exercice de l'activité $\mathrm{X}$ et les activités liées à votre processus d'insertion professionnelle?» ont été posées. La cinquième section vient clôturer l'entretien où des questions telles que «Comment s'est passé cet entretien pour vous? » et «Comment trouvez-vous ça de participer à cette recherche?» ont permis de faire un retour général sur le déroulement de l'entretien. La durée des entrevues a varié entre 111 minutes et 183 minutes et elles ont été réalisées dans une maison de transition $(n=1)$, dans un établissement d'enseignement $(n=2)$ et chez les participants $(n=2)$, dans des pièces fermées afin d'assurer la confidentialité des échanges.

Cette recherche comporte certaines limites: la taille réduite et l'absence de femmes dans l'échantillon et l'effet potentiel de désirabilité sociale associé au type de collecte de données choisi en sont les principales. À cet égard, une attention particulière a été apportée lors de l'analyse inductive délibératoire des données puisque les composantes du cadre guident l'analyse des données (Savoie-Zajc, 2004). Une 
retranscription intégrale de l'enregistrement audio des entretiens a d'abord été réalisée afin d'obtenir les échanges verbaux (Demazière et Dubar, 1997) pour une contextualisation des données, suivie d'une analyse thématique (Paillé et Mucchielli, 2012) avec le logiciel NVivo 11 selon les thèmes associés aux objectifs de recherche. Le protocole d'analyse permettait de reconstruire les composantes des activités étudiées et de répertorier les apprentissages rapportés par les participants qui ont été développés et mobilisés d'un système d'activité à un autre. Puis, des fiches synthèses ont permis une analyse transversale des données pour faire ressortir les particularités du discours des participants et relever d'autres apprentissages mobilisés qui n'avaient pas été rapportés lors des entretiens.

\section{Résultats}

Les résultats sont présentés en trois sections ${ }^{12}$. Les activités criminelles et celles liées à la recherche d'emploi sont présentées selon le modèle d'Engeström (2001). La troisième section aborde la mobilisation des apprentissages selon le concept de déplacement transfrontalier d'Engeström et al. (1995).

\section{Les activités criminelles}

Durant les entretiens, tous les participants évoquent la vente de drogue, ce qui fait référence à l'objet de l'activité.

\section{Instruments, matériels et symboliques}

Les instruments mobilisés dans la vente de drogue sont pratiquement les mêmes pour tous les participants. Les cellulaires et Facebook leur permettaient de rester en contact avec leurs clients (et avec ses employés dans le cas de Mathieu). Seuls Vincent et Mathieu avaient recours à des armes à feu pour se protéger lors des transactions.

12. En raison de la vulnérabilité associée à la population étudiée et la sensibilité du sujet de recherche, une attention particulière a été portée dans l'analyse et la présentation des résultats afin d'assurer l'anonymat des participants. Tous les prénoms utilisés sont fictifs. 
TABLEAU 1

Outils matériels

\begin{tabular}{|l|c|c|c|c|c|c|}
\hline & Christophe & Étienne & Mathieu & Vincent & William & \\
\hline Drogue & $\bullet$ & $\bullet$ & $\bullet$ & $\bullet$ & $\bullet$ & 5 \\
\hline Cellulaire & $\bullet$ & $\bullet$ & $\bullet$ & $\bullet$ & $\bullet$ & 5 \\
\hline $\begin{array}{l}\text { Balance et sacs ou } \\
\text { contenants de } \\
\text { grandeurs variables }\end{array}$ & $\bullet$ & $\bullet$ & $\bullet$ & $\bullet$ & $\bullet$ & 5 \\
\hline Automobile & $\bullet$ & $\bullet$ & $\bullet$ & & $\bullet$ & 4 \\
\hline Facebook & $\bullet$ & $\bullet$ & $\bullet$ & & $\bullet$ & 4 \\
\hline Arme à feu & & & $\bullet$ & $\bullet$ & & 2 \\
\hline & 5 & 5 & 6 & 4 & 5 & \\
\hline
\end{tabular}

TABLEA U 2

Outils symboliques

\begin{tabular}{|l|c|c|c|c|c|c|}
\hline & Christophe & Étienne & Mathieu & Vincent & William & \\
\hline Films et séries & $\bullet$ & & & $\bullet$ & $\bullet$ & 3 \\
\hline Apparence physique & $\bullet$ & & $\bullet$ & & & 2 \\
\hline Réputation & & $\bullet$ & $\bullet$ & & & 2 \\
\hline $\begin{array}{l}\text { Expérience d'autrui } \\
\text { dans le domaine }\end{array}$ & & & $\bullet$ & $\bullet$ & & 2 \\
\hline & 2 & 1 & 3 & 2 & 1 & \\
\hline
\end{tabular}

Étienne dit que ses tatouages lui facilitaient l'approche de nouveaux clients qui fréquentaient des salons de tatouage. Pour sa part, Mathieu dit que bien connaître le comportement humain l'aide à créer des liens «significatifs» auprès de futurs employés, ce qui lui vaut la réputation d'être un bon patron dans le domaine de la vente de drogue: «Faut que tu crées un lien de confiance [...], je vais le prendre comme mon chum au début $[\ldots]$ pis il vend même pas encore pour moi! Un moment donné, je lui apporte ça sur la table pis là il est total loyal envers moi!»

Comme certains l'ont déjà documenté (Akers et Jennings, 2009; Morselli et al., 2006), le mentorat et l'imitation sont là aussi mobilisés par plusieurs participants de diverses manières : certains utilisent l'expérience d'autrui dans le domaine, d'autres s'appuient sur des films et des séries pour savoir quelle attitude adopter lors d'une transaction. 
Division des tâches

Tous les participants étaient impliqués dans des réseaux de vente de drogue à différents degrés: si Christophe et Mathieu vendaient uniquement à des amis et des connaissances, Étienne, William et Vincent vendaient à un large bassin de consommateurs. Certains participants étaient associés à des groupes criminalisés et d'autres non, mais aucun ne travaillait seul. Étienne avait le plus petit réseau de l'échantillon, composé d'un fournisseur et d'un collègue. Christophe avait un collègue, un fournisseur et deux vendeurs qui travaillaient pour lui. William avait un fournisseur, un patron et un collègue. Mathieu avait sept employés et quatre fournisseurs. Vincent avait le plus grand réseau, constitué d'une trentaine de personnes: des vendeurs, des fournisseurs, des patrons et un groupe d'individus qui assuraient le lien entre les clients et les vendeurs.

\section{Communauté}

Tous les participants connaissent des personnes qui se sont impliquées dans la criminalité pour obtenir des gains financiers. Par exemple, des proches de Mathieu vendaient de la drogue et aspiraient à la même finalité: «on voulait tous se mettre millionnaires sans faire grandchose!» ou Christophe qui s'était investi dans le domaine avec son colocataire de l'époque, lorsqu'ils étaient étudiants et voulaient faire plus d'argent.

\section{Règles et croyances}

Ici, tous les participants affirment qu'il est possible de hiérarchiser les activités criminelles par leur gravité, notamment en fonction de la sentence qui y est associée. William précise par exemple qu'il est plus grave d'un point de vue juridique de vendre de la cocaïne que du cannabis. La durée de la sentence et les accusations rapportées dans le casier judiciaire associées à la vente de cocaïne ont dissuadé Christophe de s'investir dans la vente de ce produit. Concernant les règles dans l'exercice de l'activité en lien avec les forces de l'ordre, Mathieu indique: «Jamais de communication de drogue sur le téléphone dans le fond! $\mathrm{Ni}$ de quoi que ce soit d'autre qui est relié au crime...» Christophe utilisait d'ailleurs des noms de code dans son cellulaire pour joindre des clients ou ses fournisseurs. Aussi, la délation est mal vue dans le milieu. Selon Vincent, «tu fermes ta gueule devant les polices» et «tu dis jamais 
TABLEAU 3

Règles et croyances

\begin{tabular}{|c|c|c|c|c|c|c|}
\hline & Christophe & Étienne & Mathieu & Vincent & William & \\
\hline $\begin{array}{l}\text { Hiérarchiser par } \\
\text { gravité les activités } \\
\text { criminelles }\end{array}$ & $\bullet$ & $\bullet$ & $\bullet$ & $\bullet$ & $\bullet$ & 5 \\
\hline $\begin{array}{l}\text { Offrir un produit } \\
\text { de qualité }\end{array}$ & $\bullet$ & $\bullet$ & $\bullet$ & $\bullet$ & $\bullet$ & 5 \\
\hline $\begin{array}{l}\text { Prendre des } \\
\text { précautions lors de la } \\
\text { vente/de l'invasion }\end{array}$ & $\bullet$ & & $\bullet$ & $\bullet$ & $\bullet$ & 4 \\
\hline $\begin{array}{l}\text { Ne jamais parler } \\
\text { explicitement de } \\
\text { l'activité criminelle } \\
\text { par téléphone ou par } \\
\text { réseaux sociaux }\end{array}$ & $\bullet$ & $\bullet$ & $\bullet$ & $\bullet$ & & 4 \\
\hline $\begin{array}{l}\text { Ne jamais vendre } \\
\text { chez soi pour } \\
\text { préserver son } \\
\text { intimité }\end{array}$ & & $\bullet$ & $\bullet$ & & $\bullet$ & 3 \\
\hline $\begin{array}{l}\text { Ne pas vendre } \\
\text { directement dans } \\
\text { les bars }\end{array}$ & $\bullet$ & & $\bullet$ & & $\bullet$ & 3 \\
\hline $\begin{array}{l}\text { Toujours en référer à } \\
\text { son patron en cas } \\
\text { de conflits avec des } \\
\text { clients ou d'autres } \\
\text { vendeurs }\end{array}$ & $\bullet$ & & & $\bullet$ & $\bullet$ & 3 \\
\hline \multirow{2}{*}{$\begin{array}{l}\text { Vendre chez soi pour } \\
\text { éviter de se faire voir } \\
\text { par la police }\end{array}$} & $\bullet$ & & & • & & 2 \\
\hline & 7 & 4 & 6 & 6 & 6 & \\
\hline
\end{tabular}

le nom du boss». Concernant le rapport aux autres acteurs du milieu criminel, William précise que «les bars, ça appartient tout le temps à quelqu'un [...] qu'on appelle qui fait du corner, fait qu'il se tient au bar pis lui sa job c'est de vendre...» et qu'il est dangereux de vendre sur le territoire d'un autre vendeur. À l'inverse, Étienne ne se souciait pas des territoires ni ne prenait de précautions particulières, ce qui a pu expliquer le fait qu'il se soit fait arrêter selon lui. Pour plusieurs, il faut toujours en référer à son patron lorsqu'il y a une mésentente avec un autre vendeur ou un client et éviter de se faire justice soi-même. William spécifie: «Quelqu'un vient me faire des menaces, je vais pas le cogner 
tout de suite parce que tu sais pas, il est backé par quelqu'un, il travaille pour quelqu'un [...]. Appelle ton boss, il va appeler son boss, pis c'est là que ça va se régler.» Finalement, concernant le rapport à la clientèle, tous tenaient à vendre un produit de qualité qui n'était pas transformé ou dilué pour éviter les commentaires de clients insatisfaits.

\section{Résultats}

Pour tous les participants, la retombée centrale dans la vente de drogue est l'argent. Plusieurs s'y sont investis, d'abord pour rentabiliser leur consommation, et en faire ensuite une activité professionnelle. Outre cette retombée, une forme de reconnaissance symbolique et des apprentissages réalisés ont été rapportés comme bénéfices de l'exercice de ces activités. Finalement, se retrouver avec un casier judiciaire est une retombée involontaire chez tous les participants.

TABLEA U 4

Objets liés à l'exercice d'activités criminelles

\begin{tabular}{|l|c|c|c|c|c|c|}
\hline & Christophe & Étienne & Mathieu & Vincent & William & \\
\hline $\begin{array}{l}\text { Obtenir des gains } \\
\text { financiers }\end{array}$ & $\bullet$ & $\bullet$ & $\bullet$ & $\bullet$ & $\bullet$ & 5 \\
\hline $\begin{array}{l}\text { Être bon vendeur et } \\
\text { créer des liens } \\
\text { facilement avec les } \\
\text { autres }\end{array}$ & $\bullet$ & $\bullet$ & $\bullet$ & $\bullet$ & $\bullet$ & 5 \\
\hline $\begin{array}{l}\text { Aptitudes en calcul } \\
\text { mental }\end{array}$ & $\bullet$ & & $\bullet$ & $\bullet$ & $\bullet$ & 4 \\
\hline $\begin{array}{l}\text { Offrir un bon service } \\
\text { à la clientèle }\end{array}$ & $\bullet$ & & $\bullet$ & $\bullet$ & $\bullet$ & 4 \\
\hline $\begin{array}{l}\text { Capacité d'analyse et } \\
\text { d'observation }\end{array}$ & & $\bullet$ & $\bullet$ & & $\bullet$ & 3 \\
\hline $\begin{array}{l}\text { Reconnaissance } \\
\text { symbolique }\end{array}$ & & $\bullet$ & $\bullet$ & $\bullet$ & $\bullet$ & 5 \\
\hline $\begin{array}{l}\text { Obtention d'un casier } \\
\text { judiciaire }\end{array}$ & $\bullet$ & 4 & 7 & 6 & 7 & \\
\hline
\end{tabular}

La section suivante concerne le deuxième objectif qui est de relever et de décrire les activités liées à la recherche d'emploi. 


\section{Les activités liées à la recherche d'emploi}

$\mathrm{Au}$ moment des entretiens, Étienne est travailleur autonome, Christophe travaille dans le secteur de la réfrigération et William fait des inventaires pour des entreprises. Vincent et Mathieu sont en recherche d'emploi. Pour chacun, trouver des employeurs potentiels et leur faire parvenir leur curriculum vitæ sont deux étapes qui ont été sélectionnées d'emblée et retenues comme objets dans la modélisation de l'activité d'Engeström.

\section{Instruments matériels et symboliques}

Presque tous les participants mobilisent leur cellulaire, leur curriculum vitæ et la section Placement en ligne du site d'Emploi-Québec. La manière de faire de Christophe est représentative de l'ensemble des participants: «Je vais sur Emploi-Québec, je vais voir les emplois, mettons je marque serveur, OK. Tous les restaurants sont affichés, OK parfait! Je prends lui, lui, lui, lui. Je me lève, prends mon char, je m'en vais au lieu puis je vais rencontrer le gérant.» De manière générale, les participants semblent plus enclins à recourir à des instruments matériels numériques comme le cellulaire et l'ordinateur, comparativement aux instruments papier (bottin

TA B LE A U 5

Outils matériels

\begin{tabular}{|l|c|c|c|c|c|c|}
\hline & Christophe & Étienne & Mathieu & William & Vincent & \\
\hline Curriculum vitæ & $\bullet$ & $\bullet$ & $\bullet$ & $\bullet$ & $\bullet$ & 5 \\
\hline $\begin{array}{l}\text { Site de placement en } \\
\text { ligne d'Emploi-Québec }\end{array}$ & $\bullet$ & $\bullet$ & $\bullet$ & $\bullet$ & $\bullet$ & 5 \\
\hline Cellulaire & $\bullet$ & $\bullet$ & $\bullet$ & $\bullet$ & $\bullet$ & 5 \\
\hline Automobile & $\bullet$ & $\bullet$ & & $\bullet$ & & 3 \\
\hline Site de jobboom & & & & $\bullet$ & $\bullet$ & 2 \\
\hline Site de Facebook & $\bullet$ & $\bullet$ & & & & 2 \\
\hline Site de Google & & & $\bullet$ & $\bullet$ & & 2 \\
\hline Journaux et affiches & & & $\bullet$ & & $\bullet$ & 2 \\
\hline Site de Blackmarket & & & & & $\bullet$ & 1 \\
\hline Site de Kijiji & & & & & $\bullet$ & 1 \\
\hline Site de Jobillico & & & & $\bullet$ & & 1 \\
\hline Bottin téléphonique & & & $\bullet$ & & & 1 \\
\hline & 5 & 5 & 6 & 7 & 7 & \\
\hline
\end{tabular}


téléphonique et journaux) que seuls Vincent et Mathieu utilisent. Plus spécifiquement, Vincent dit trouver des emplois en se promenant et en voyant des affiches qui demandent du personnel.

TABLEAU 6

Outils symboliques

\begin{tabular}{|l|c|c|c|c|c|c|}
\hline & Christophe & Étienne & Mathieu & Vincent & William & \\
\hline Réseau de contacts & $\bullet$ & & $\bullet$ & & $\bullet$ & 3 \\
\hline CJE & $\bullet$ & & $\bullet$ & $\bullet$ & $\bullet$ & 4 \\
\hline Centre local d'emploi & & & $\bullet$ & $\bullet$ & $\bullet$ & 3 \\
\hline & 2 & 0 & 3 & 2 & 3 & \\
\hline
\end{tabular}

Le réseau de contacts est nommé d'entrée de jeu par tous, à l'image de Mathieu: «C'est surtout ça qui m'aide dans le fond, parce que j'ai une grosse famille... Pis dans chacun de leur domaine, ils sont capables tout le temps de me placer en quelque part...» Les organismes en employabilité sont également reconnus comme des outils de grande importance. Vincent affirme ainsi qu'«au CJE ${ }^{13}$, [ils] sont 50000 à vouloir m'aider...». Et bien que Christophe, William et Mathieu ne sollicitent pas ces organismes, ils les nomment néanmoins comme ressources potentielles.

\section{Division des tâches}

Pour les participants, les intervenants des organismes communautaires, des carrefours jeunesse-emploi (CJE) et des centres locaux d'emploi constituent des ressources importantes ou du moins accessibles dans leur recherche d'emploi. Par ailleurs, les participants mentionnent les membres de leur réseau social comme des ressources qui les informent des milieux en recherche de personnel pour les aiguiller dans leurs démarches.

13. Les Carrefours Jeunesse Emploi (CJE) sont des organismes d'aide à l'emploi et à l'intégration sociale dédiés aux jeunes adultes. 


\section{Communauté}

À l'exception d'Étienne, qui a cessé de fréquenter des gens du milieu criminel, tous déclarent connaître plusieurs personnes ayant un casier judiciaire qui tentent de s'insérer sur le marché du travail. Vincent affirme que les gens qu'il connaît dans cette situation rencontrent de grandes difficultés à s'insérer. Cela pourrait expliquer le manque de motivation et le pessimiste ressentis quant à sa recherche d'emploi au moment de l'entretien. La communauté, en tant que composante de l'activité, ne semble pas contribuer à priori à la réalisation de l'activité liée à leur recherche d'emploi.

TABLEA U 7

Règles et croyances

\begin{tabular}{|c|c|c|c|c|c|c|}
\hline & Christophe & Étienne & Mathieu & William & Vincent & \\
\hline $\begin{array}{l}\text { Accès refusé à } \\
\text { certains domaines } \\
\text { avec un casier } \\
\text { judiciaire }\end{array}$ & $\bullet$ & $\bullet$ & $\bullet$ & $\bullet$ & $\bullet$ & 5 \\
\hline Postuler en personne & $\bullet$ & $\bullet$ & $\bullet$ & & $\bullet$ & 4 \\
\hline $\begin{array}{l}\text { Éviter d'informer } \\
\text { l'employeur du casier } \\
\text { judiciaire }\end{array}$ & & & $\bullet$ & $\bullet$ & $\bullet$ & 3 \\
\hline $\begin{array}{l}\text { Informer l'employeur } \\
\text { du casier judiciaire }\end{array}$ & $\bullet$ & $\bullet$ & & & & 2 \\
\hline \multirow[t]{2}{*}{ Postuler en ligne } & & & & $\bullet$ & & 1 \\
\hline & 3 & 3 & 3 & 3 & 3 & \\
\hline
\end{tabular}

Règles et croyances

À l'exception d'Étienne, tous déclarent qu'il est difficile, voire impossible, d'accéder à certains métiers et formations avec un casier judiciaire. Christophe, qui aurait aimé être infirmier, précise: «J'ai un dossier criminel pour possession dans le but de faire le trafic [...] même si j'aurais le goût de me trouver une job dans un hôpital, ça arrivera peutêtre pas!» Mathieu mentionne que les secteurs où les conditions sont difficiles (travailler à l'extérieur, lever des charges lourdes, travailler sur de longues périodes) sont plus accessibles avec un casier alors qu'«au McDo, lui, c'est le contraire! [...] Tout le monde est capable de la faire la job, fait qu'on veut un gars qui est top clean, qui a pas de dossier». 
Selon lui, «il y a des restrictions, là j'ai un dossier on se mentira pas! Il y a des jobs que je peux pas faire». Vincent évite d'aller porter son c.v. dans des entreprises qui font affaire avec les États-Unis, car «aussitôt que tu as un dossier, tu peux pas rentrer là!». Pour lui, il semble plus facile de réinvestir les activités criminelles que de s'insérer sur le marché du travail: «Pour ça qu'il y a beaucoup de récidivisme, le monde se dit: «Ça donne rien de chercher de la job, on recommence à vendre!»» Tous appréhendent le moment où un employeur leur demandera s'ils ont un casier judiciaire. À l'exception d'Étienne, dont les nombreux tatouages apparents pourraient amener les employeurs à éliminer sa candidature, casier judiciaire ou pas. Le casier judiciaire agit comme un stigmate invisible qui devient visible à partir du moment où un employeur pose la question. Selon qu'il soit visible ou pas, le stigmate va influencer les stratégies ou comportements de la personne qui le porte (Goffman, 1963): on le voit bien ici pour Étienne qui est le seul à ne pas appréhender ce moment car il est le seul à porter un stigmate visible.

\section{Résultats}

Pour tous les participants, les retombées associées au fait de chercher un emploi semblent être de rencontrer un employeur et d'obtenir une entrevue d'embauche. Toutefois, lors de ces entrevues, mentionner le casier judiciaire peut rebuter certains employeurs. La mobilisation de certains apprentissages réalisés dans des activités criminelles au regard du concept du déplacement transfrontalier pourrait avoir un impact sur l'entrevue d'embauche. La section suivante porte sur le troisième objectif qui est de cibler des apprentissages réalisés dans l'exercice d'activités criminelles mobilisés dans leurs recherches d'emploi.

\section{Le déplacement transfrontalier}

Tous les participants affirment que le fait d'avoir un casier judiciaire influence leur recherche d'emploi. En effet, si ceux-ci arrivent à réaliser certaines activités (trouver des employeurs potentiels et leur faire parvenir leur curriculum vitæ), il leur est difficile d'obtenir un emploi une fois que l'employeur apprend qu'ils ont un casier judiciaire. Vincent, qui n'a pas d'emploi au moment de l'entretien, dit que «si tu as un dossier, souvent ils vont mettre ton nom en bas de la pile...» et précise «quand tu cherches une job pis ils voient que tu as un dossier, ils vont dire: «Vous 
êtes trop qualifié» ou ils vont te dire une autre excuse bâtarde pour pas dire que c'est à cause que tu as un dossier». Mathieu, en recherche d'emploi, affirme: «[L'employeur] va checker mon dossier criminel, il va faire: «Oh shit, je le veux pas ici!»». Pour William, qui travaille pour une compagnie d'inventaire, «une [employeuse] m'a dit justement: «Tu as déjà fait un crime, moi qu'est-ce qui va me dire que tu en feras pas d'autres?», j'ai répondu: «Si j'avais voulu déjà faire d'autres crimes, je chercherais pas une job payée à 10,75 de l'heure...»». Au regard des difficultés d'insertion en raison notamment d'un casier judiciaire, mobiliser - consciemment ou non - certains apprentissages développés dans des activités criminelles pourrait parfois compenser leur manque d'expérience, pallier leur apparence physique et atténuer la gravité de leur casier judiciaire pour se démarquer en entrevue d'embauche.

Les participants nomment certains apprentissages réalisés dans la vente de drogue qui influencent leur manière de se vendre en entrevue. Parfois, la mobilisation est effectuée dans des contextes jugés similaires aux deux activités analysées. Étienne, travailleur autonome dans le commerce au détail, dit avoir appris à être un bon vendeur en vendant de la drogue: «d'une manière ou d'une autre, j'étais dans le domaine de la vente! J'étais dans le domaine du public. Fait que ça m’a apporté des compétences dans ce milieu-là...». Cela lui sert dans son insertion professionnelle pour se vendre en entrevue et précise être désormais un excellent vendeur. William se vend en entrevue avec plus d'assurance depuis ses activités criminelles: «j'ai eu une certaine confiance en moi aussi en vendant de la coke ${ }^{14} \ldots »$ et précise qu'avec cette confiance en soi découlant de son statut de vendeur de drogue (forme de reconnaissance symbolique), il n'hésitera pas à argumenter les avantages d'être embauché auprès d'un employeur, ce qu'il ne faisait pas avant. Vincent mentionne qu'il serait bon pour vendre du cannabis à des fins médicales: «je connais le milieu [...] je connais le monde [...] auquel ça entoure pis ça, ça rattache» et qu'il pourrait mobiliser ses apprentissages en vente dans des contextes similaires, mais légaux. Pour Christophe, dans le secteur de la réfrigération, bien connaitre son produit et prendre le temps de le décrire à ses clients pour le vendre est quelque chose qu'il a appris dans la vente de drogue. Il semble mobiliser cet apprentissage en entrevue, en se décrivant d'une manière spécifique: "J'ai jamais eu de problème avec les entrevues là! [...] Je suis préparé, je sais

14. Terme faisant référence à la cocaïne. 
ce que j'ai à dire... Je me connais, je sais comment que je vais être, je sais comment agir, j'ai mes qualités, mes défauts, je suis capable de les dire...» Pour Mathieu (en recherche d'emploi), la mobilisation des apprentissages se réalise aussi dans des contextes considérés sensiblement identiques (vente illicite et vente licite): «juste la clientèle elle change pis les fournisseurs changent, mais c'est quand même acheter des trucs pour les revendre plus cher...». Il mobilise des apprentissages réalisés dans des activités criminelles dans celles liées à sa recherche d'emploi: «c'est la même affaire que de vendre de la drogue, je vais convaincre le gars que mon produit il est mieux, il est de meilleure qualité pis que personne va y faire ça». Cela rappelle sa manière de se vendre en entrevue en montrant qu'il est indispensable et qu'il est l'employé recherché: «Faut que tu saches parler [...] faut que tu ailles l'air motivé [...]. Faut que tu te rendes indispensable!» Ces apprentissages peuvent contribuer à le démarquer en entrevue et faire bonne impression auprès de l'employeur, malgré son casier judiciaire. Ledit casier, en tant qu'obstacle à l'insertion professionnelle, peut être considéré ici comme une contradiction nuisant à la réalisation des activités de recherche d'emploi. Pour tenter de résoudre ou de contourner cette contradiction, les participants mobilisent alors des apprentissages réalisés dans des activités criminelles; un travail de mobilisation qui illustre empiriquement le concept de déplacement transfrontalier.

Il est également possible de cerner certains apprentissages réalisés dans la vente de drogue que les participants considèrent en mesure de mobiliser dans des contextes cette fois-ci assez différents. William (en emploi) dit: «j'aurais été excellent pour un métier de livraison! [...], je la connais ta ville, pis je sais qui habite où [...] pis en plus, je compte vite avec les sous! C'est des trucs justement que... Tu peux pas mettre ça dans ton CV! Pourtant ce l'est, c'est vraiment quelque chose que tu as appris [...], un travail d'infirmière tu as besoin d'être bonne sous pression [...], mais si tu as vendu de la drogue, t'as été sous pression crissement souvent!» Pour Vincent (en recherche d'emploi), il semble que d'autres processus de mobilisation apparemment plus implicites soient à l'œuvre. L'analyse amène à voir qu'il utilise sa capacité d'analyse développée dans la vente de drogue pour se distinguer en entrevue: «Mettons [que] je suis au Tim Hortons ${ }^{15}$ pis je vais checker, je vais étudier les personnes: [elles] ont l'air à être rapides, dynamiques, tout ça... Bien

15. Chaîne de restauration rapide. 
tsé, je dis: «Je suis rapide, dynamique».» Il semble en mesure de cibler les informations qu'un employeur désire entendre en entrevue, ce qui pourrait l'aider à se démarquer. Même au sein de contextes à priori différents, les participants effectuent un travail de réflexion de la mobilisation de leurs apprentissages pour résoudre certaines contradictions se présentant dans leurs activités de recherche d'emploi.

\section{Conclusion}

Les résultats posent à nouveau la question de la reconnaissance des apprentissages réalisés dans des contextes variés, bien au-delà de la formation formelle (Delors, 1996). Ce qui soulève un paradoxe: d'un côté le discours social insiste sur la nécessité de disposer de qualifications formelles (Champagne et al., 2013), alors que selon Martucelli (2009), il importe de considérer l'individu dans toutes ses pluralité, diversité et dispositions. En cela, la reconnaissance des apprentissages réalisés dans des activités criminelles pourrait contribuer plus largement aux efforts liés à la réinsertion sociale des personnes judiciarisées.

Une première étape dans ce processus de reconnaissance pourrait s'effectuer chez les personnes intervenantes (spécialistes en orientation, criminologues, travailleurs sociaux, etc.) qui agissent en première ligne à titre de ressources nommées et mobilisées par les participants. Cela interpelle également les champs d'intervention dédiés à cette population, car des approches compartimentées par discipline sont susceptibles de constituer un obstacle à la reconnaissance de ces apprentissages, surtout lorsqu'ils se sont développés dans des activités sur lesquelles pèse encore une forte stigmatisation sociale (Roy et Hurtubise, 2004) - mais qui parallèlement se rapprochent étroitement de compétences valorisées en employabilité (savoir se vendre auprès d'un employeur, démontrer une confiance en soi, utiliser sa capacité d'analyse pour cibler ce que l'employeur recherche, etc.) (Morselli et Tremblay, 2004).

Le concept de déplacement transfrontalier offre des avenues prometteuses en matière d'intervention. À partir d'un objectif d'emploi établi avec la personne judiciarisée, il est possible d'explorer les contradictions potentielles empêchant son atteinte, dont le casier judiciaire, tel que le rapporte Pager (2003) dans une recherche se penchant sur les contrecoups du casier judiciaire sur l'employabilité. Solliciter les apprentissages issus des activités criminelles comme ressources potentielles pour résoudre ou contourner ces contradictions, notamment en ce qui 
concerne le casier judiciaire, peut s'avérer fécond comme le montrent nos résultats. Ces avenues de réflexion pourraient, ultimement, avoir un effet potentiel, sur l'insertion et le maintien des personnes judiciarisées sur le plan social et professionnel et contribuer à la diminution des risques de récidive. Finalement, sans normaliser ou cautionner les actes criminels commis, la reconnaissance par les personnes intervenantes de ces apprentissages - sans réduire les personnes judiciarisées à ces seuls apprentissages - comme leviers potentiels devant les contradictions rencontrées dans des activités de recherche d'emploi, pourrait contribuer à rehausser leur sentiment d'efficacité personnelle relativement à leurs démarches et à développer un regard moins normatif sur leurs propres expériences et apprentissages.

Par ailleurs, Engeström considère les activités comme des construits dynamiques. À cet égard, la mobilisation des apprentissages est un processus tout aussi dynamique et notre appareillage méthodologique n'a saisi qu'une partie des apprentissages réalisés et mobilisés au regard de la réappropriation de leur histoire (Demazière, 2007) qu'en avaient les participants au moment de l'entretien ${ }^{16}$. Cette recherche contribue à documenter l'un des résultats associés aux activités criminelles, soit la réalisation d'apprentissages, considérant le peu d'écrits les concernant. Notre recherche illustre certaines contradictions et enjeux spécifiques auxquels font face des jeunes adultes judiciarisés dans leurs tentatives d'insertion (Comité consultatif clientèle judiciarisée adulte, 2017) et de conceptualiser comment certains apprentissages réalisés dans les activités criminelles ont une influence sur des activités liées à la recherche d'emploi (Morselli et Tremblay, 2004). Mais, se pencher plus spécifiquement sur les parcours biographiques et leurs ruptures dans l'acquisition et le développement d'apprentissages et de compétences permettrait d'alimenter les connaissances sur le sujet (Ouellet et Bouchard, 2017).

Dans le même sens, des recherches ultérieures qui se pencheraient sur d'autres activités criminelles que celles répertoriées ici, sur l'expérience de femmes judiciarisées ou encore sur l'ajustement des interventions au regard de cette reconnaissance par exemple, contribueraient à cet effort scientifique et social mené dans plusieurs champs discipli-

16. Les apprentissages étudiés se sont probablement construits aussi dans d'autres activités que celles criminelles, notamment en milieu scolaire, ce qui contribue à leur caractère dynamique. 
naires d'accompagner plus adéquatement ces populations vulnérables sur qui pèse souvent un regard normatif.

\section{Références}

Adler, P. A. et Adler, P. (1983). Shifts and oscillations in deviant careers: The case of upper-level drug dealers and smugglers. Social Problems, 31(2), 195207.

Akers, R. L. et Jennings, W. G. (2009). Social learning theory. Dans J. Miller (dir.), 21st Century criminology: A reference bandbook (p. 323-332). Thousand Oaks, CA: SAGE Publications.

Bakx, A., Bakker, A., Koopman, M. et Beijaard, D. (2016). Boundary crossing by science teacher researchers in a PhD program. Teaching and Teacher Education, 60, 76-87.

Becker, H. (1985). Outsiders. Paris, France: Métailié.

Blumstein, A. et Cohen, J. (1987). Characterizing criminal careers. Science, 237(4818), 985-991.

Bureau, S. et Fendt, J. (2010). L'entrepreneuriat au sein de l'économie informelle des pays développés: une réalité oubliée? Dans Actes de la $19^{\circ}$ Conférence de l'AIMS, Luxembourg, 1er-4 juin 2010. Repéré à www.strategie-aims.com/ events/conferences/2-xixeme-conference-de-1-aims/communications/13lentrepreneuriat-au-sein-de-leconomie-informelle-des-pays-developpesune-realite-oubliee/download

Champagne, A., Malenfant, R., Bellemare, G. et Briand, L. (2013). L'intégration des jeunes peu qualifiés dans une entreprise d'économie sociale: travailler autrement? Reflets: revue d'intervention sociale et communautaire, 19(1), 185223.

Charest, M. et Tremblay, P. (2009). Immobilité sociale et trajectoires de délinquance. Revue française de sociologie, 50(4), 693-718.

Charette, Y. (2010). La perception du prestige des occupations illicites par des délinquants: une perspective sur les capitaux criminels (Mémoire de maîtrise, Université de Montréal). Repéré à https://papyrus.bib.umontreal.ca/xmlui/ handle/1866/4551

Cohen, A. K. (1965). The sociology of the deviant act: Anomie theory and beyond. American sociological review, 30(1), 5-14.

Comité consultatif clientèle judiciarisée adulte. (2014). Clientèle judiciarisée: son profil et ses besoins. Repéré à www.cccja.org/wp-content/ uploads/2016/06/Profil-clientele-judiciarisee.pdf

Comité consultatif clientèle judiciarisée adulte. (2017). Les personnes judiciarisées, un bassin de travailleurs pour le Québec. Repéré à http://www.cccja. org/wp-content/uploads/2017/08/CCCJA-les-personnes-judiciaris \% C3\% A9es-un-bassin-de-travailleurs-pour-le-Quebec-1.pdf

Côté, P.-B., Blais, M., Bellot, C. et Manseau, H. (2013). Des expériences affectives et sexuelles en situation de rue. Criminologie, 46(2), 243-261. 
Delors, J. (dir.). (1996). L'éducation, un trésor est caché dedans. Rapport à l'UNESCO de la Commission internationale sur l'éducation pour le vingt et unième siècle. Paris, France: Éditions Odile Jacob.

Demazière, D. (2007). Quelles temporalités travaillent les entretiens biographiques rétrospectifs? Bulletin de méthodologie sociologique, 93. Repéré à bms. revues.org/index 506.html

Demazière, D. et Dubar, C. (1997). Analyser les entretiens biographiques: l'exemple de récits d'insertion. Paris, France: Nathan.

Dionne, P. (2015). Le groupe d'insertion sociale et professionnelle: apprentissages et développement au cour de l'activité collective de personnes en situation de chômage de longue durée (Thèse de doctorat, Université de Sherbrooke). Repéré à https://savoirs.usherbrooke.ca/handle/11143/7552

Engeström, Y. (2001). Expansive learning at work: Toward an activity theoretical reconceptualization. Journal of Education and Work, 14(1), 133-156.

Engeström, Y. (2005). Developmental work research: Expanding activity theory in practice. Berlin, Allemagne: Lehmanns Media.

Engeström, Y., Engeström, R. et Kärkkäinen, M. (1995). Polycontextuality and boundary crossing in expert cognition: Learning and problem solving in complex work activities. Learning and Instruction, 5, 319-336.

Engeström, Y. et Sannino, A. (2011). Discursive manifestations of contradictions in organizational change efforts. A methodological framework. Journal of Organizational Change Management, 24(3), 368-389.

Engeström, Y. et Sannino, A. (2013). La volition et l'agentivité transformatrice: perspective théorique de l'activité. Revue internationale du CRIRES: innover dans la tradition de Vygotsky, 1(1), 4-19.

Fortin, M.-F. (2010). Fondements et étapes du processus de recherche: méthodes quantitatives et qualitatives ( $2^{\mathrm{e}}$ éd.). Montréal, Québec: Chenelière Éducation.

Fouquet, A. (2011). Travail, emploi, activité - Une histoire sociale. Annales des Mines-Réalités Industrielles, 1, 11-15.

Goffman, E. (1963). Stigmate. Les usages sociaux des handicaps (traduit par A. Kihm). Les Éditions de Minuit.

Gouvernement du Québec. (2011). Plan stratégique 2011-2014. Québec, Québec: Ministère de l'Emploi et de la Solidarité sociale. Repéré à http://www.mess. gouv.qc.ca/publications/pdf/ADMIN_Plan_Strategique_2011-2014.pdf

Lanctôt, N. (2005). Liens entre l'inadaptation scolaire des adolescents et des adolescentes judiciarisés et leur adaptation sociale et personnelle à l'âge adulte. Nouveaux c@hiers de la recherche en éducation, 8(2),89-100.

Le Blanc, M. (1986). La carrière criminelle: définition et prédiction. Criminologie, 19(2), 79-99.

Le Blanc, M. (2003). La conduite délinquante des adolescents: son développement et son explication. Dans M. Le Blanc, M. Ouimet et D. Szabo (dir.), Traité de criminologie empirique ( $3^{\mathrm{e}}$ éd., p. 367-420). Montréal, Québec: Les Presses de l'Université de Montréal.

Le Blanc, M. (2010). Un paradigme développemental pour la criminologie: développement et autorégulation de la conduite déviante. Criminologie, 43(2), 401-428. 
Letkemann, P. (1973). Crime and work. Englewood Cliff, NJ: Prentice Hall.

Loughran, T. A., Nguyen, H., Piquero, A. R. et Fagan, J. (2013). The returns to criminal capital. American Sociological Review, 78, 925-948.

Martuccelli, D. (2009). Qu'est-ce qu'une sociologie de l'individu moderne? Pour quoi, pour qui, comment? Sociologies et société, 41(1), 15-33.

McCarthy, B. et Hagan, J. (2001). When crime pays: Capital, competence and criminal success. Social Forces, 79, 1035-1059.

McCarthy, B. et Hagan, J. (2004). L'argent change tout: les revenus personnels des adolescents et leur penchant à la délinquance. Criminologie, 37(2), 123149.

McCarthy, B., Hagan, J. et Cohen, L. (1998). Uncertainty, cooperation, and crime: Understanding the decision to co-offend. Social Forces, $77(1), 155-$ 184.

Morselli, C. et Tremblay, P. (2004). Délinquance, performance et capital social: une théorie sociologique des carrières criminelles. Criminologie, 37(2), 89-122.

Morselli, C., Tremblay, P. et McCarthy, B. (2006). Mentors and criminal achievement. Criminology, 44(1), 17-43.

Ouellet, F. (2012). La dynamique des séquences de rechute chez des joueurs excessifs et des joueurs délinquants. Criminologie, 45(2), 51-85.

Ouellet, F. et Bouchard, M. (2017). Only a matter of time? The role of criminal competence in avoiding arrest. Justice Quarterly, 34(4), 699-726.

Pager, D. (2003). The mark of criminal record. American Journal of Sociology, 108(5), 937-975.

Piquero, A., Farrington, D. et Blumstein, A. (2003). The criminal career paradigm. Crime and Justice, 30, 359-506.

Paillé, P. et Mucchielli, A. (2012). L'analyse qualitative en sciences bumaines et sociales. ( $3^{e}$ éd.). Paris, France: Armand Colin.

Robitaille, C. (2004). À qui profite le crime? Les facteurs individuels de la réussite criminelle. Criminologie, 37(2), 33-62.

Roy, S. et Hurtubise, R. (2004). De l'itinérance et du travail. À propos de la reconnaissance sociale des pratiques dans l'univers de la marginalité. Dans J. Poupart (dir.), Au-delà du système pénal. L'intégration sociale et professionnelle des groupes judiciarisés et marginalisés (p. 125-140). Québec, Québec: Presses de l'Université du Québec.

Savoie-Zajc, L. (2004). La recherche qualitative/interprétative en éducation. Dans T. Karsenti et L. Savoie-Zajc (dir.), La recherche en éducation: étapes et approches (p. 123-150). Sherbrooke, Québec: Éditions du CRP.

Savoie-Zajc, L. (2009). L'entrevue semi-dirigée. Dans B. Gauthier (dir.), Recherche sociale. De la problématique à la collecte de données (5éd., p. 337-360). Québec, Québec: Presses de l'Université du Québec.

Sutherland, E. H. (1966). Principes de criminologie. Paris, France: Édition CUJAS. Tremblay, P. (2010). Le délinquant idéal. Performance, discipline, solidarité. Montréal, Québec: Liber. 
Tremblay, P. et Morselli, C. (2000). Patterns in criminal achievement: Wilson and Abrahamse revisited. Criminology, 38, 633-657.

Uggen, C. et Thompson, M. (2003). The socioeconomic determinants of illgotten gains: Within-person changes in drug use and illegal earnings. American Journal of Sociology, 109(1), 146-185.

Vitaro, F., Brendgen, M., Ladouceur, R. et Tremblay, R. E. (2001). Gambling, delinquency, and drug use during adolescence: Mutual influences and common risk factors. Journal of Gambling Studies, 17(3), 171-190.

Vultur, M., Trottier, C. et Gauthier, M. (2002). Les jeunes Québécois sans diplôme. Perspectives comparées sur l'insertion professionnelle et le rapport au travail. Dans D.-G. Tremblay et L. F. Dagenais (dir.), Ruptures, segmentations et mutations du marché du travail (p. 71-94). Sainte-Foy, Québec: Presses de l'Université du Québec.

Warr, M. (1998). Life-course transitions and desistance from crime. Criminology, $36(2), 183-216$.

\title{
Benefits of crime: Learnings aquired through criminal activities incorporated into the professionnal integration process
}

\begin{abstract}
Criminal activities are part of that category of social life activities that is subject to stigmatization. However, a number of studies in criminology show that criminals benefit from such activities in terms of financial gains, recognition, and knowledge. While some research discusses the knowledge needed to perform criminal activities, few articles consider the knowledge gained through such activities, some of which could provide valuable skills for socially valued employment if it were not for social attitudes toward them. Incorporating knowledge learned while practicing criminal activities into the process of integration into a profession would better recognize the complexity of this process, particularly for young adults, with or without diplomas. This paper shows the result of qualitative research with five young adults who had been involved with criminal activities to see if the knowledge they had acquired could be incorporated into their integration into a legal job.
\end{abstract}

KEYWORDS - Young adults, knowledge, criminality, professional integration, activity theory.

\section{Cuando el crimen paga. Los aprendizajes realizados en actividades criminales movilizados en el proceso de inserción profesional}

RESUMEN - Las actividades criminales hacen parte de la categoría de actividades de la vida social que tienen una cierta estigmatización. Sin embargo, algunas investigaciones en criminología han demostrado que las personas que ejercen estas actividades obtienen beneficios: beneficios financieros, reconocimiento simbólico y aprendizajes variados. Si bien algunas investigaciones exponen los aprendizajes realizados para poder ejercer estas actividades criminales, pocas se interesan a los aprendizajes reali- 
zados en la práctica de estas actividades. Sin embargo, algunos de estos aprendizajes serían competencias socialmente valorizadas en lo referente a la empleabilidad si no fuera por el juicio social que pesa sobre ellos. Algunos aprendizajes realizados en el ejercicio de actividades criminales, movilizados en el proceso de inserción profesional, ofrecerían una comprensión más justa de la complejidad del proceso de inserción profesional, sobre todo de los adultos jóvenes, ya sean diplomados o no. Este artículo presenta los resultados de una investigación cualitativa, conducida con cinco adultos jóvenes que habían ejercido actividades criminales, para ver los aprendizajes movilizados, o susceptibles de serlo, en el proceso de inserción profesional en un empleo legal.

PALABRAS CLAVE • Jóvenes adultos, aprendizajes, criminalidad, inserción profesional, teoría de la actividad. 